\title{
Thermal Oxidation Stability of Interesterified Oils under Continuous Heating Conditions
}

\author{
Myoung-Ae Kim, ${ }^{*}$ Teruyoshi Matoba and Kiyozo Hasegawa \\ Department of Food Science and Nutrition, \\ *Graduate Division of Human Culture, Nara Women's University, \\ Nara 630, Japan \\ Received December 7, 1987
}

\begin{abstract}
Mixed oils and the corresponding interesterified oils of soybean, palm and coconut were investigated as to their thermal deterioration during continuous heating at $180^{\circ} \mathrm{C}$. The extent of thermal deterioration of each oil increased in proportion to the degree of unsaturation, there being nearly no difference at all between the mixed oils and the corresponding interesterified oils. However, the refractive index of the interesterified oils increased more rapidly than that of the others. We supposed that the rapid increase in this value in the case of interesterifed oils may be caused by an increase in the total number of triacylglycerol molecules, which were composed of easily oxidizable unsaturated and fairly stable saturated fatty acids, resulting in polymerization.
\end{abstract}

Interesterification is important as a means of reforming triacylglycerols in edible oils. Through its use, fats and oils of higher quality, as to not only physical properties, ${ }^{1,2)}$ but also nutritive value ${ }^{3,4)}$ and stability, can be 'tailor made'. It has been reported that the influence of interesterification on the stability, as to autoxidation, differs with the oil. ${ }^{5 \sim 8}$ The influence of interesterification on the stability during thermal oxidation, on the other hand, has not yet been elucidated. The authors previously reported that the characteristic foaming of a mixture of lauric and nonlauric oils under deep-fat frying conditions was eliminated on interesterification. ${ }^{\text {) }}$

This paper deals with the influence of interesterification on the thermal oxidation stability of mixed oils during continuous heating at high temperature, using soybean (Soy), palm (Palm) and coconut (Coco) oils, whose fatty acid compositions differ from each other.

\section{MATERIALS AND METHODS}

Preparation of sample oils. As single oils, refined Soy, Coco (Nakarai Chemicals Ltd.) and Palm (Yamakei Sangyo Ltd.) were used. The mixed oils (M) were $1: 1$ (w/w) mixtures of Soy and Coco, and Soy and Palm, respectively. The interesterified oils (E) were obtained by random interesterification of the respective mixed oils, with $1 \% \mathrm{NaOCH}_{3}$ as a catalyst, at $80^{\circ} \mathrm{C}$ for $30 \mathrm{~min}^{8,10}$ Each single oil, mixed oil or interesterified oil was purified by passage through a Florisil (Wako Pure Chemicals Industry Ltd.) column, and then the triacylglycerol fraction obtained was used as the sample oil for the following experiments.

Heating conditions and sampling. Twenty-five grams of a sample oil in a test tube $(20 \mathrm{~cm} \times 27 \mathrm{~mm}$ i.d. $)$ was heated continuously in a silicone oil bath at $180^{\circ} \mathrm{C}$ for $50 \mathrm{hr}$. The heating was conducted in the presence of air without stirring. An aliquot $(600 \sim 700 \mathrm{mg}$ each $)$ was collected from the center of the residual oil at regular intervals and stored under $\mathrm{N}_{2}$ gas at $-22^{\circ} \mathrm{C}$ until analyses. New oil was not added after each sampling.

Analytical methods. The absorption at $232 \mathrm{~nm}\left(A_{232}\right)$ was measured for a $0.33 \%$ solution of a sample oil diluted with isooctane. ${ }^{11,12)}$ The peroxide value (PV) was determined by the method of Asakawa and Matsushita. ${ }^{13}$ The carbonyl value (CV) and acid value (AV) were determined by micro methods in the usual way. ${ }^{14,15}$ The refractive index (RI) was measured at $44^{\circ} \mathrm{C}$ with an Abbe refractometer. ${ }^{16)}$

Fatty acid compositions of heated oils. Methyl esters of fatty acids solely of the ester form in acylglycerols were obtained by base-catalyzed transesterification. ${ }^{17,18)}$ The fatty acid compositions (FA) were determined by gas- 
liquid chromatography according to the method of Daun and Mazur, ${ }^{19)}$ using a Shimadzu GC-9A gas chromatograph equipped with a flame ionization detector and a glass column $(2.5 \mathrm{~m} \times 3 \mathrm{~mm}$ i.d.) packed with $3 \% \mathrm{SP}-2310 / 2 \%$ SP-2300 on Chromosorb W AW (100 200 mesh; Supelco, Inc., Bellefonte, PA). The flow rate of nitrogen gas was $60 \mathrm{ml} / \mathrm{min}$, and the column oven temperature was programmed from 100 to $200^{\circ} \mathrm{C}$ at the rate of $5^{\circ} \mathrm{C} / \mathrm{min}$. Margaric acid methyl ester was used as an internal standard.

Triacylglycerol compositions. The triacylglycerol compositions were determined gas-liquid chromatographically by carbon number (CN) analysis, in which the total number of carbon atoms in the fatty acid moiety was analyzed. Gas-liquid chromatography for $\mathrm{CN}$ analysis was carried out with a Shimadzu GC-9A gas chromatograph equipped with a flame ionization detector and a glass column $(0.5 \mathrm{~m} \times 3 \mathrm{~mm}$ i.d.) packed with Dexsil $300 \mathrm{GC}$ on Chromosorb W AW (60 80 mesh; Shinwa Chemical Industries Ltd., Kyoto). The column oven temperature was programmed from $250^{\circ} \mathrm{C}$ to $365^{\circ} \mathrm{C}$ at a rate of $5^{\circ} \mathrm{C} / \mathrm{min}$

\section{RESULTS}

The thermal oxidation during continuous high temperature heating was followed by means of measurements of $A_{232}, \mathrm{PV}, \mathrm{CV}, \mathrm{AV}$, $\mathrm{FA}$, and RI, and the following results were obtained.

\section{Absorbance at $232 \mathrm{~nm}$}

The $A_{232}$ values for Soy, M-Soy-Palm, MSoy-Coco, E-Soy-Palm and E-Soy-Coco increased with the heating time (Fig. 1). The $A_{232}$ value increase rate for Soy was the highest, and those for the mixed and interesterified oils reflected the mix ratio at $50 \%$ in each increase rate of $A_{232}$ value. Coconut oil showed a very low $A_{232}$ response. In the case of Soy-Palm, the $A_{232}$ value increase rates for the mixed and interesterified oils were almost the same. In the case of Soy-Coco, the $A_{232}$ value increase rate for the interesterified oil was higher than that for the mixed oil.

\section{Peroxide value}

Except for in the case of Coco, PVs remained below 4 (Fig. 2). PV of Coco increased rapidly to 14 on heating for 6 to $15 \mathrm{hr}$, and decreased gradually thereafter. Although both

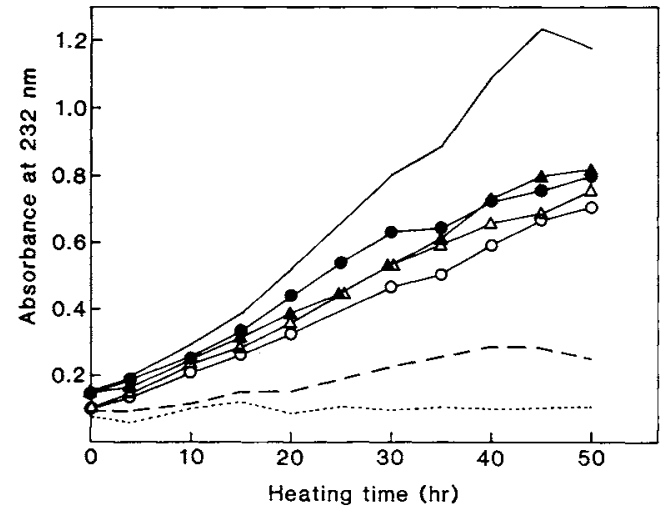

FIG, 1. UV Absorbance at $232 \mathrm{~nm}$ of Each Oil with Heating Time during Thermal Oxidation.

The sample oils were diluted in isooctane to a final concentration of $3.33 \%$. Soy, _-; Palm, ---; Coco, --.--; M-Soy-Palm, $\triangle-\triangle$; M-Soy-Coco, O-O; E-SoyPalm, $\boldsymbol{\Delta} \mathbf{\Delta}$; E-Soy-Coco,

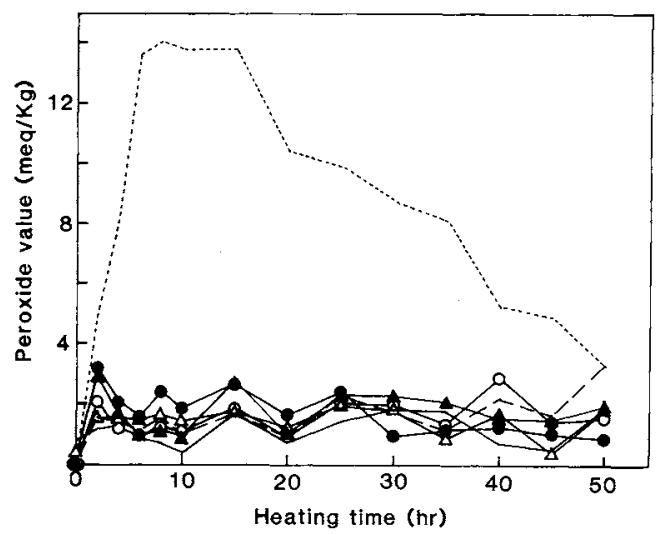

FIG. 2. Effect of Heating Time on the Peroxide Values of Oils.

The symbols are the same as in Fig. 1.

M-Soy-Coco and E-Soy-Coco included 50\% Coco, these oils did not show a rapid increase in PV.

\section{Carbonyl value}

All the sample oils showed a linear increase in $\mathrm{CV}$ with heating time (Fig. 3). The times taken to reach CV 40 were estimated to be as follows; $20 \mathrm{hr}$ for Soy, $40 \mathrm{hr}$ for Coco, $50 \mathrm{hr}$ for Palm, and 25 to $30 \mathrm{hr}$ for M-Soy-Palm, MSoy-Coco, E-Soy-Palm and E-Soy-Coco. The $\mathrm{CV}$ increase rate for Soy was the highest, $\mathrm{CV}$ being more than 100 after $50 \mathrm{hr}$-heating. The 


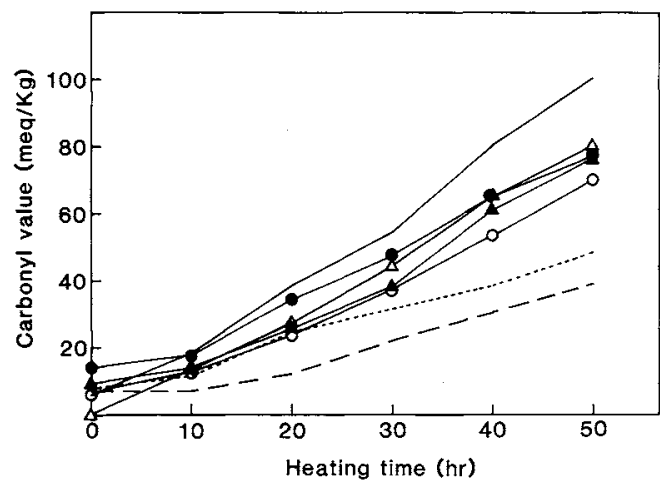

FIG. 3. Effect of Heating Time on the Carbonyl Values of Oils.

The symbols are the same as Fig. 1.

$\mathrm{CV}$ increase rate for M-Soy-Palm was higher than that for E-Soy-Palm.

\section{Acid value}

Figure 4 shows the changes in $\mathrm{AV}$ of the sample oils during thermal oxidation. The acid value of Coco markedly increased with heating time, and after $50 \mathrm{hr}$-heating it had increased to 1.7 , while those of the other oils increased only 0.4 to 0.7 . The AV increase rate for Coco was delayed on mixing or interesterification with $50 \%$ Soy. Interesterification hardly affected the change in $A V$, and the AV increase rate for E-Soy-Coco was a little higher than that for M-Soy-Coco.

\section{Fatty acid compositions}

Table I shows the deterioration degree based on the fatty acid composition of and the percentage of the residual fatty acids in each oil after $0 \mathrm{hr}, 20 \mathrm{hr}$ and $40 \mathrm{hr}$-heating. For all the sample oils, the rates of decrease in unsaturated fatty acids were higher than in the cases of saturated acids, in the order of linolenic $>$ linoleic $>$ oleic acid. The linoleic acid content mainly influenced the percentage of the total amount of residual fatty acids in each oil. After $40 \mathrm{hr}$-heating, the percentages of residual fatty acids were in the range of 65 to $80 \%$ for all the sample oils, Coco showing the highest percentage, $80.5 \%$.

On the other hand, the rates of decrease in

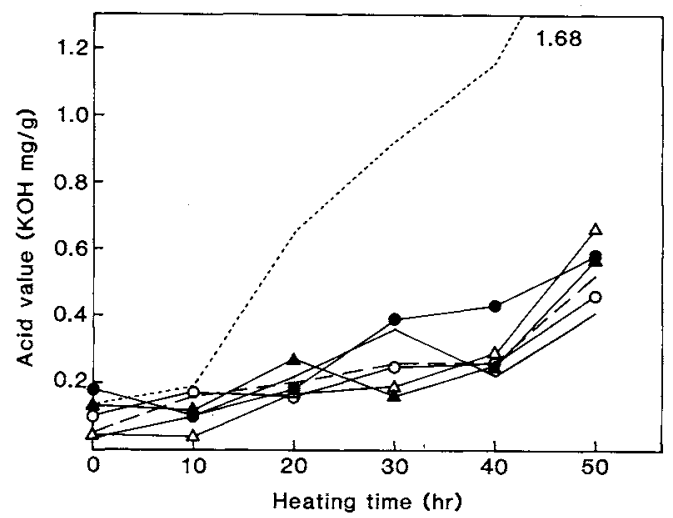

FIG. 4. Effect of Heating Time on the Acid Values of Oils.

The symbols are the same as in Fig. 1.

oleic, linoleic and linolenic acids for M-SoyPalm were higher than those for E-Soy-Palm. For Soy-Coco, the rates of decrease in unsaturated fatty acids did not differ between MSoy-Coco and E-Soy-Coco. But, the rates of decrease in saturated fatty acids were higher for E-Soy-Coco than for M-Soy-Coco, in particular, the decrease in lauric acid being high in absolute amount.

\section{Refractive index}

Every oil showed an increase in RI with heating time (Fig. 5). The RI of each mixed oil at $0 \mathrm{hr}$-heating was a value which reflected those of the component oils in a mix ratio of $50 \%$. The RI increase rates for the mixed oils reflected those of the component oils in a similar manner. However, RIs for the two interesterified oils increased rapidly with heating time. The reason why this unusual increase was found for the interesterified oils will be discussed below.

\section{DISCUSSION}

During thermal oxidation, the unsaturated fatty acids formed actively conjugated dienes, ${ }^{11,12,20)}$ and then the hydroperoxides formed from unsaturated acids at that time were decomposed faster than those formed from saturated fatty acids. ${ }^{21)}$ In this study, it 
Table I. Changes in Fatty Acid Composition during Thermal Oxidation

\begin{tabular}{|c|c|c|c|c|c|c|c|c|c|c|c|}
\hline \multirow{2}{*}{ Heated oils } & \multirow{2}{*}{ Hours } & \multicolumn{10}{|c|}{ Fatty acid $(\%)$} \\
\hline & & $8: 0$ & $10: 0$ & $12: 0$ & $14: 0$ & $16: 0$ & $18: 0$ & $18: 1$ & $18: 2$ & $18: 3$ & Total \\
\hline \multirow{3}{*}{ Soy } & 0 & & & & & 10.0 & 3.9 & 25.7 & 53.9 & 6.5 & 100.0 \\
\hline & 20 & & & & & 10.0 & 3.6 & 23.9 & 36.9 & 3.0 & 77.4 \\
\hline & 40 & & & & & 10.2 & 3.6 & 22.4 & 26.8 & 1.6 & 64.6 \\
\hline \multirow{3}{*}{ Palm } & 0 & & & & 0.9 & 44.3 & 5.0 & 40.6 & 9.2 & & 100.0 \\
\hline & 20 & & & & 0.8 & 41.4 & 4.0 & 33.8 & 5.0 & & 85.0 \\
\hline & 40 & & & & 0.8 & 42.0 & 4.1 & 29.4 & 2.7 & & 79.0 \\
\hline \multirow{3}{*}{ Coco } & 0 & 7.2 & 5.9 & 48.4 & 18.2 & 8.9 & 2.7 & 6.9 & 1.8 & & 100.0 \\
\hline & 20 & 7.4 & 5.8 & 47.5 & 17.5 & 8.6 & 2.6 & 3.9 & & & 93.3 \\
\hline & 40 & 6.5 & 5.0 & 41.7 & 15.2 & 7.6 & 2.6 & 1.9 & & & 80.5 \\
\hline \multirow{3}{*}{$\mathrm{M}^{a}$-Soy-Palm } & 0 & & & & 0.5 & 26.8 & 4.0 & 33.0 & 32.5 & 3.2 & 100.0 \\
\hline & 20 & & & & 0.4 & 25.5 & 3.9 & 28.7 & 20.3 & 1.4 & 80.2 \\
\hline & 40 & & & & 0.4 & 24.9 & 3.8 & 24.9 & 11.9 & 0.5 & 66.4 \\
\hline \multirow{3}{*}{ M-Soy-Coco } & 0 & 2.7 & 2.3 & 21.1 & 7.9 & 9.9 & 3.4 & 17.6 & 31.5 & 3.6 & 100.0 \\
\hline & 20 & 2.8 & 2.2 & 18.9 & 7.3 & 8.8 & 3.2 & 14.3 & 18.3 & 1.4 & 77.2 \\
\hline & 40 & 3.1 & 2.3 & 19.9 & 7.6 & 9.4 & 3.1 & 13.2 & 11.6 & 0.6 & 70.8 \\
\hline \multirow{3}{*}{$\mathrm{E}^{b}$-Soy-Palm } & 0 & & & & 0.5 & 27.5 & 4.1 & 33.2 & 31.5 & 3.2 & 100.0 \\
\hline & 20 & & & & 0.4 & 25.1 & 3.8 & 28.9 & 20.5 & 1.4 & 80.1 \\
\hline & 40 & & & & 0.4 & 26.4 & 4.1 & 27.4 & 13.4 & 0.6 & 72.3 \\
\hline \multirow{3}{*}{ E-Soy-Coco } & 0 & 3.3 & 2.9 & 24.2 & 8.9 & 9.7 & 3.2 & 16.2 & 28.3 & 3.3 & 100.0 \\
\hline & 20 & 2.6 & 2.0 & 18.7 & 8.1 & 9.7 & 3.3 & 14.4 & 17.1 & 1.3 & 77.2 \\
\hline & 40 & 3.0 & 2.1 & 18.2 & 7.2 & 9.0 & 3.1 & 12.3 & 10.3 & 0.5 & 65.7 \\
\hline
\end{tabular}

a. Mixed, $50: 50(\mathrm{w} / \mathrm{w})$.

$b$ Interesterified.

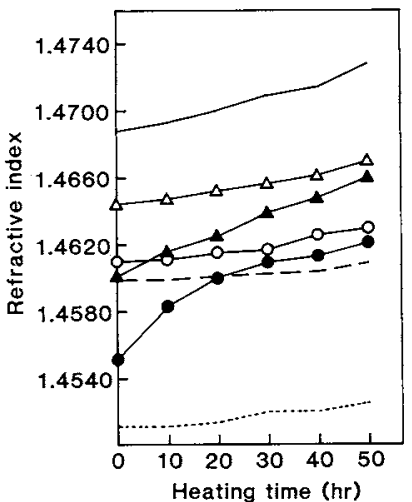

FIG. 5. Changes in the Refractive Indexes of Oils with Heating Time, $0 \sim 50 \mathrm{hr}$.

The symbols are the same as in Fig. 1.

was found that the higher the content of unsaturated fatty acids of each oil was, the higher the rate of increase in $A_{232}$ or CV became (Figs. 1 and 3).
Every oil showing high rates of increase in $A_{232}$ and $\mathrm{CV}$ had a low PV, below 4, for all heating times. ${ }^{11,20 \sim 22)}$ At high temperature, saturated fatty acids were able to form hydroperoxides which were not liable to decompose thermally. ${ }^{23,24)}$ In the present study, the behavior of Coco as to PV was rather peculiar (Fig. 2). PV of Coco increased up to 14 on heating for 6 to $15 \mathrm{hr}$. This might mean that saturated fatty acids of medium chain length, the main constituents of Coco, formed hydroperoxides which were somewhat more stable than those formed from unsaturated fatty acids. However, M-Soy-Coco and E-Soy-Coco did not show a tendency of a rapid increase in $\mathrm{PV}$, in spite of the presence of $50 \%$ Coco (Fig. $2)$. The reason for this suppression is considered to be that the unsaturated fatty acids may undergo oxidative decomposition more preferentially when unsaturated and saturated 
fatty acids are present simultaneously. An alternative interpretation is considered to be that if hydroperoxides were formed from the saturated fatty acids of Coco, those hydroperoxides would be liable to decomposition by radicals derived from the oxidized unsaturated fatty acids of Soy at $50 \%$, as a result of which, a remarkable increase in PV was not observed.

Coco showed a particularly high AV increase rate, the rate significantly decreasing on mixing or interesterification with $50 \%$ Soy (Fig. 4). Yuki ${ }^{25)}$ reported that epoxy compounds retarded the hydrolysis of oils. The retardation of the AV increase for the mixed and interesterified oils described above seemed to be due to epoxy compounds derived from the unsaturated fatty acids of Soy.

The change in the FA of each oil showed that the unsaturated fatty acids were very unstable during the thermal oxidation. The total contents of residual fatty acids were influenced mainly by the rate of decrease in the content of unsaturated fatty acids (Table I). The rates of decrease in oleic, linoleic and linolenic acids for M-Soy-Palm were greater than those for E-Soy-Palm. This result may be related to the higher $\mathrm{CV}$ increase rate for $\mathrm{E}$ Soy-Palm. Also, in the case of Soy and Coco, it can be assumed that the higher $\mathrm{AV}$ increase rate for the interesterified oil than for the mixed oil is closely associated with the higher rates of decrese in saturated fatty acids such as lauric or myristic acid in the interesterified oil.

The influence of interesterification on the thermal oxidation of oils was hardly detected on chemical analyses, as described above. Rapid increases were observed, however, on RI analyses of the interesterified oils (Fig. 5). Thermal oxidative polymerization has been reported to be first induced by the formation of conjugated dienes. ${ }^{26)}$ The extents of formation of conjugated dienes for the mixed and interesterified oils were similar to each other, as shown by the fact that no difference was observed in the changes in $A_{232}$ (Fig. 1). Increases in RIs of oils during thermal oxidation have been reported by several investigators. ${ }^{16,27)}$ Generally, polymerization causes
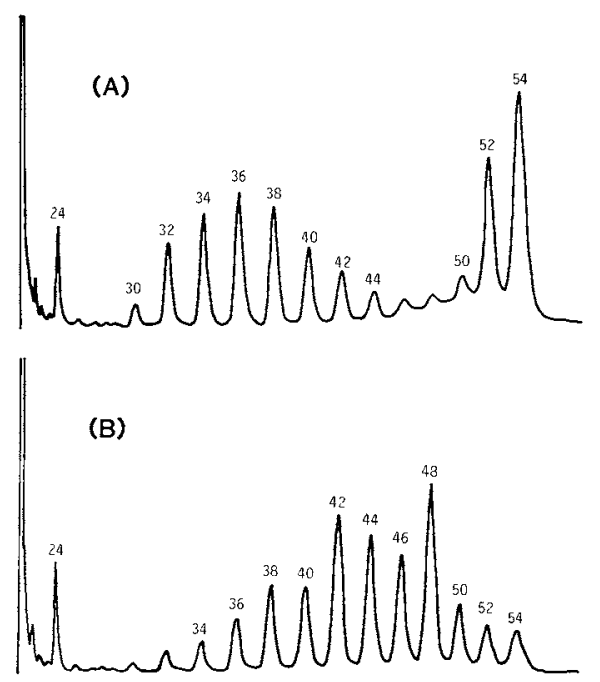

FIG. 6. Changes in the Carbon Number of Soybean Oil Mixed with Coconut Oil before and after Interesterification.

(A), before interesterification; (B), after interesterification.

an increases in RI. ${ }^{28)}$ In this study, the RI increase rates for interesterified oils were higher than those for the mixed oils, while the mixed and interesterified oils had the same FAs (Table I). The triacylglycerol molecules of the two interesterified oils in this study were mainly composed of unsaturated and saturated fatty acids. ${ }^{9}$ The changes in $\mathrm{CNs}$ for Soy-Coco before and after interesterification are shown in Fig. 6 as representative results. The triacylglycerols of CN 42 and CN 48 of ESoy-Coco were formed through interesterification from those of $\mathrm{CN} 32 \sim 40$ of Coco and those of CN $50 \sim 54$ of Soy. It was considered that the triacylglycerols of $\mathrm{CN} 42$ were formed from $\mathrm{C} 12: 0 \times 2$ and $(\mathrm{C} 18: 1$ or $\mathrm{C} 18: 2) \times 1$, and those of $\mathrm{CN} 48$ from $\mathrm{C} 12: 0 \times 1$ and $(\mathrm{C} 18: 1$ or $\mathrm{C} 18: 2) \times 2$, or $\mathrm{C} 12: 0 \times 1, \mathrm{C} 18: 1 \times 1$ and $\mathrm{C} 18: 2$ $\times 1$, respectively, because the triacylglycerols of CN50 54 consisted mainly of unsaturated fatty acids, i.e., oleic and linoleic acids, according to the FA results in Table $I$. That is, the total number of triacylglycerol molecules with one or two unsaturated fatty acids increased with interesterification. This type of triacylglycerol is easily oxidizable, accompanying with a stability due to the pres- 
ence of saturated acyl moieties. Such molecules are liable to polymerize and the polymerized compounds are fairly stable. Consequently, it is considered that the RIs of the interesterified oils increased rapidly, and the RI increase rates for these oils were also higher than that for Soy.

Studies on the effect of interesterification on autoxidation suggested that the rate of autoxidation of an interesterified oil is influenced by the binding position of unsaturated fatty acids in the triacylglycerol. ${ }^{5 \sim 8)}$ In this study, on the contrary, it was found that the stability, on the thermal oxidation of oils, was not influenced by the interesterification reaction under the extreme conditions of a high temperature, but by the fatty acid composition. However, only a rapid increase in RI occurred for an interesterified oil with triacylglycerol consisting of unsaturated and saturated fatty acids.

Acknowledgments. The authors wish to thank Dr. A. Okumura, Professor of the Faculty of Science, Nara Women's University, for the measurement of and discussion on the refractive index. This study was supported by a Grant-in-Aid for Scientific Research (No. 61580064) from the Ministry of Education, Science and Culture of Japan, and by the Foundation for Promotion of Food Sciences and Technology.

\section{REFERENCES}

1) S. J. Laning, J. Am. Oil Chem. Soc., 62, 400 (1985).

2) N. Matsui, Yukagaku, 28, 680 (1979).

3) S. Mukheerjee and S. Sengupta, J. Am. Oil Chem. Soc., 58, 287 (1981).

4) S. Adhikari, J. Dasgupta, D. Bhattachauyya and M. M. Chakrabarty, Fette Seifen Anstrichm., 83, 345 (1981).

5) K. G. Raghuveer and E. G. Hammond, J. Am. Oil Chem. Soc., 44, 239 (1967).

6) F. Y. Lau, E. G. Hammond and P. F. Ross, J. Am. Oil Chem. Soc., 59, 407 (1982).

7) S. Wada and C. Koizumi, Yukagaku, 35, 549 (1986).
8) S. Wada and C. Koizumi, J. Am. Oil Chem. Soc., 60 1105 (1983).

9) M. A. Kim, T. Matoba and K. Hasegawa, Agric. Biol. Chem., 52, 693 (1988).

10) D. K. Park, J. Terao and S. Matsushita, Agric. Biol. Chem., 47, 121 (1983).

11) L. M. Du Plessis, J. Am. Oil Chem. Soc., 58, 5785 (1981).

12) S. H. Yoon, S. K. Kim, M. G. Shin and K. H. Kim, J. Am. Oil Chem. Soc., 62, 1485 (1985).

13) T. Asakawa and S. Matsushita, Lipids, 15, 965 (1980).

14) S. Ohta, "Deterioration of Fried Food and Its Protection," Saiwai Shobo, Tokyo, 1977, pp. $224 \sim 225$.

15) Y. Fujino, "An Introduction to Lipid Analysis," Biochemical Experimental Method Series, No. 9, Gakkai Shuppan Center, Tokyo, 1980, pp. 51 52.

16) S. Ohta and E. Yuki, "Theory and Practice of Fried Food," Saiwai Shobo, Tokyo, 1976, pp. 380 381.

17) T. Yamakawa, in "Chemistry of Lipids," Biochemical Experimental Course Series, Vol. 3, ed. by the Japanese Biochemical Society, Tokyo-KagakuDojin, Tokyo, 1974, p. 199.

18) W. W. Christie, "Lipid Analysis," 2nd Ed., Pergamon Press, Oxford, 1982, pp. 53 54.

19) J. K. Daun and P. B. Mazur, J. Am. Oil Chem. Soc., 60, 1751 (1983).

20) M. Peled, T. Gutfinger and A. Letan, J. Sci. Food Agric., 26, 1655 (1975)

21) E. Yuki and K. Morimoto, Yukagaku, 31, 915 (1982)

22) C. W. Fritsch, D. C. Egberg and J. S. Magnuson, J. Am. Oil Chem. Soc., 56, 746 (1979).

23) H. S. Pardun and J. Blass, Fette Seifen Anstrichm., 76, 97 (1974).

24) T. Kawada and M. Yamazaki, Yukagaku, 20, 295 (1971).

25) E. Yuki, Yukagaku, 16, 660 (1967).

26) M. W. Formo, in "Bailey's Industrial Oil and Fat Products," Vol. 1, 4th Ed., ed. by D. Swern, John Wiley \& Sons, Inc., New York, 1979, pp. 217 221, $698 \sim 701$.

27) B. Toi and S. Ohta, Kaseigaku-Zasshi, 8, 269 (1957).

28) S. Okamura, "Experimental Chemistry Course Series," Vol. 6, ed. by Chemical Society of Japan, Maruzen, Tokyo, 1956, pp. 206 207. 